\title{
HYPERPLASIA OF THE HYPOPHYSIS CEREBRI?
}

\author{
BY \\ J. D. Cummins \\ DUBLIN
}

ENDOCRINE therapy is, for the most part, still in an empirical stage. That there is a chemical inter-relation between the organs, having an internal secretion, one cannot doubt. And so, in vague morbid states, attributed to disharmony between these organs, endocrine therapy is employed, with the hope of restoring the normal harmonious relationships. Of the endocrine preparations, thyroid extract is, perhaps, most frequently used in this way. Sometimes the effect is very gratifying; at other times no apparent improvement takes place. The following case is one of the gratifying ones, and has certain features of interest.

An unmarried woman of 33 had been complaining of progressive failure of vision during three months For a year she had been under the care of a physician for amenorrhoea, and during that time had become very stout and lethargic. If the patient sat down to read or sew she often fell asleep. Previously the patient had led an active life, and, but for suppurating cervical glands in childhood, had never been ill.

Examination of the eyes: R.V. 1/60, L.V. 6/18.

Bitemporal hemianopia-no error of refraction-Wernicke's pupillary reaction not obtained-discs hyperaemic, otherwise fundi normal.

Gynaecological examination : Uterus small, in proper position -no pathological evidence in pelvic viscera.

X-ray report. No enlargement of pituitary fossa-contours of sella Turcica and clinoid processes well-defined and regular. Wassermann reaction negative.

Urine free from sugar and albumin-no polyuria.

This clinical complex recalled some of the concomitants of pregnancy - the amenorrhoea, the increase of weight (over and above the contents of the gravid uterus), the bitemporal contraction of the visual fields, the hypertrophy of the hypophysis. The thyroid gland may show a swelling during pregnancy and at each menstrual period. And, in congenital aplasia of the thyroid, an enlargement of the hypophysis takes place (Zuckermann).

In dystrophia adiposa genitalis, dependent upon disease of the anterior lobe of the hypophysis, there is an excessive deposit of adipose tissue throughout the body, with involution of the genitalia. 
Of particular interest, in my case, were the impairment of vision . and, especially, the hemianopic fields, which provided a reliable means of observing the effects of treatment, which consisted of one grain of thyroid extract daily by the mouth.

There was a rapid improvement in the right eye, visual acuity increased from $1 / 60$ to $6 / 60$, and the field showed a definite enlargement in nine,days. The improvement continued, without interruption, until full vision and a full field were regained by each eye. The dates on the accompanying charts mark the rate of improvement.

As I hinted in my opening paragraph, the administration of thyroid extract was empirical. I hoped, by daily repeating a small dose, to coax back into agreement the hypothetically disordered relations between the hormone-producing organs. It was a lucky hit. But how was the bitemporal hemianopia to be explained? And why was the vision of the right eye more impaired than of the left? The conjecture, which I am about to offer, may appear less fanciful, if it is borne in mind that there was no clinical evidence of increased intra-cranial pressure; that there was no enlargement of the pituitary fossa or indistinctness of the clinoid processes; and, finally, that the efficacy of the treatment manifested itself promptly.

I conceived that a hyperplasia of the anterior lobe of the hypophysis formed a mass, which protruded through the top of the sella Turcica and inclined towards the right, rather than straight forwards towards the chiasma. Then, either by pressure, partly on the chiasma and partly on the right optic tract, or by toxaemia (as I think more likely) analogous to that which may bring about retrobulbar neuritis in sphenoidal empyema, the hypothetical mass determined the characters of the fields and the unequal impairment of central vision.

Menstruation recurred five months after treatment had been begun. Two months later the regular rhythm of the function was restored. Treatment was discontinued. There has been no recurrence of the symptoms.

While under treatment, a record was made at regular intervals of the patient's weight. To her great surprise there was no progressive diminution in weight, although four months of treatment had necessitated the "taking in" of her various garments. "It is certain that I am getting thinner," wrote the patient, and then, somewhat petulantly, "I suppose I'm becoming more solid."

The charts represent the fields of a $3 \mathrm{~mm}$. white spot. On January 10, 1922, there was an absolute central scotoma for red, green and blue. Peripheralwards the colours were recognized. 
In the right eye, peripheral colour perception was confined to the upper nasal quadrant.

As the patient lived 90 miles from Dublin, and as travelling was a somewhat adventurous affair, the number of visits was few and but little time was available for plotting out colour fields.

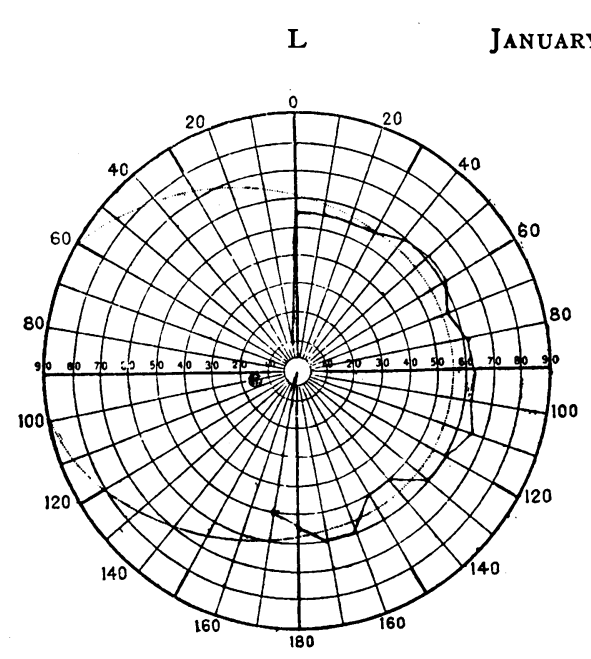

Visual Acuity-6/18.

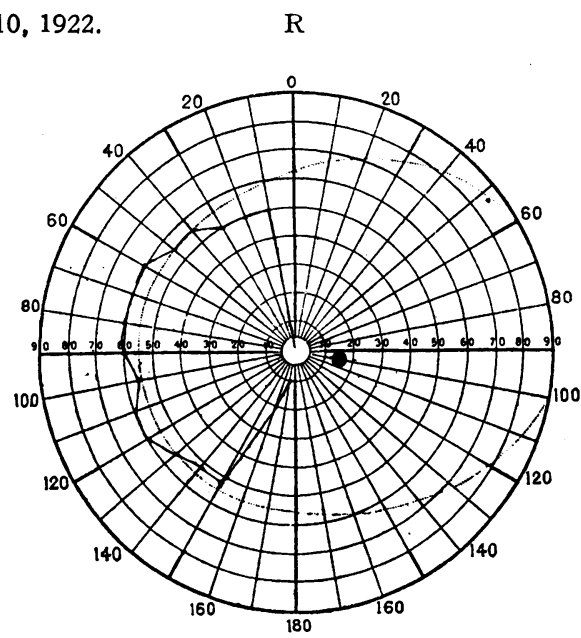

Visual Acuity $-1 / 60$.

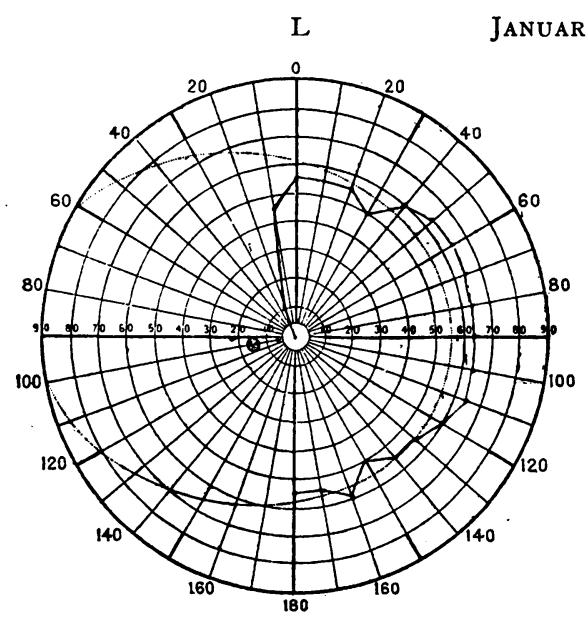

Visual Acuity-6/12.

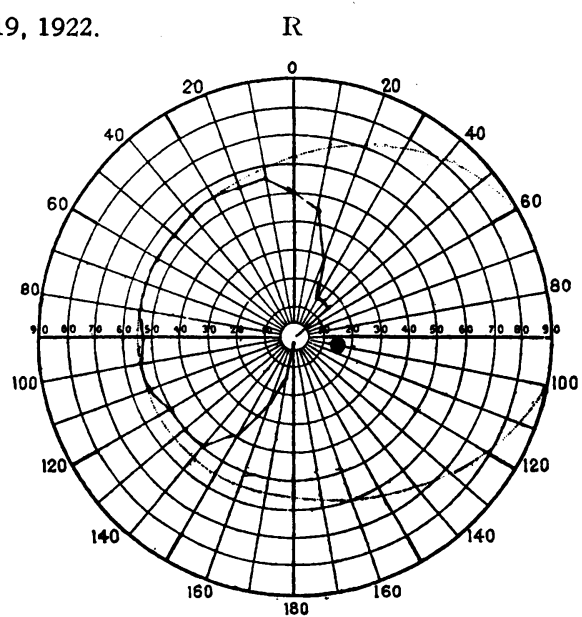

Visual Acuity $-6 / 60$. 


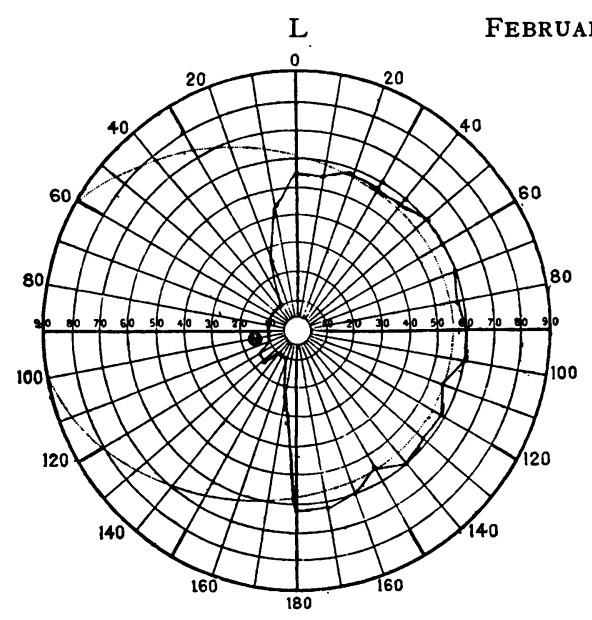

Visual Acuity $-6 / 12$.

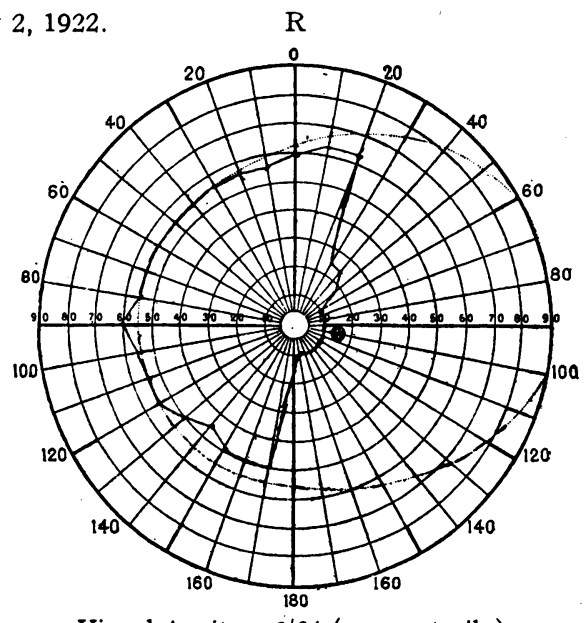

Visual Acuity-6!24 (momentarily).

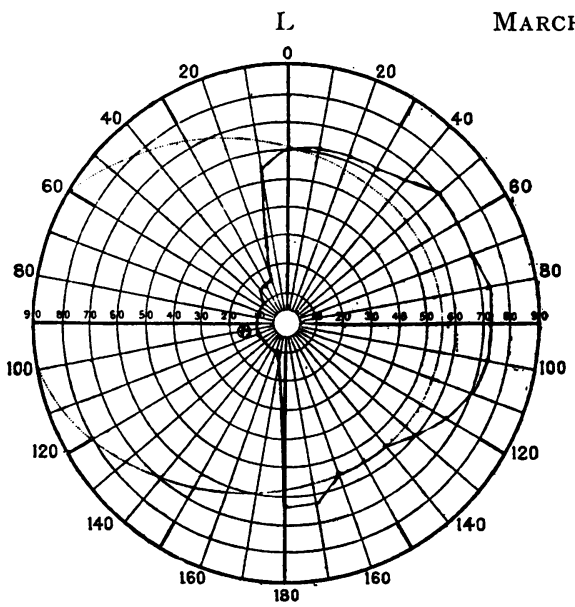

Visual Acuity-6/12.

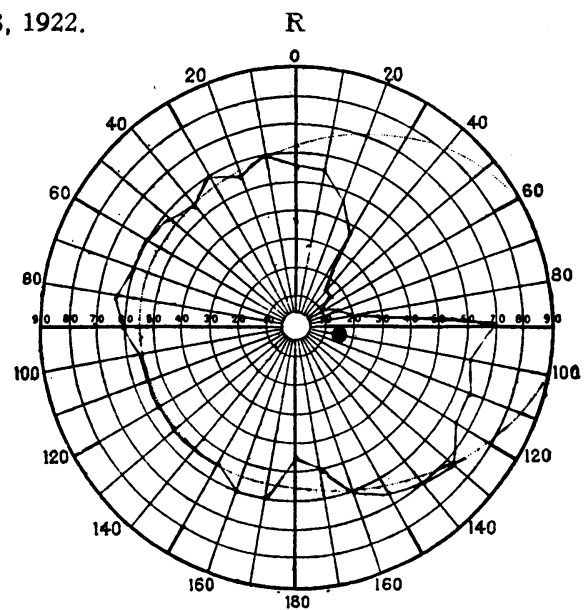

Visual Acuity $-6 / 24$.

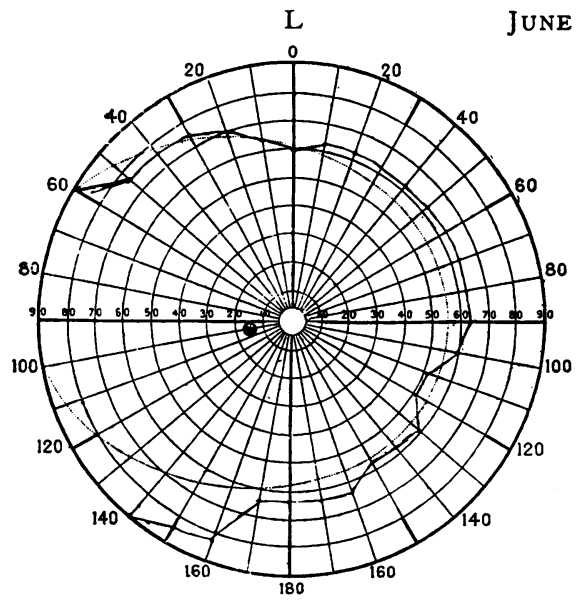

Visual Acuity-6/6.

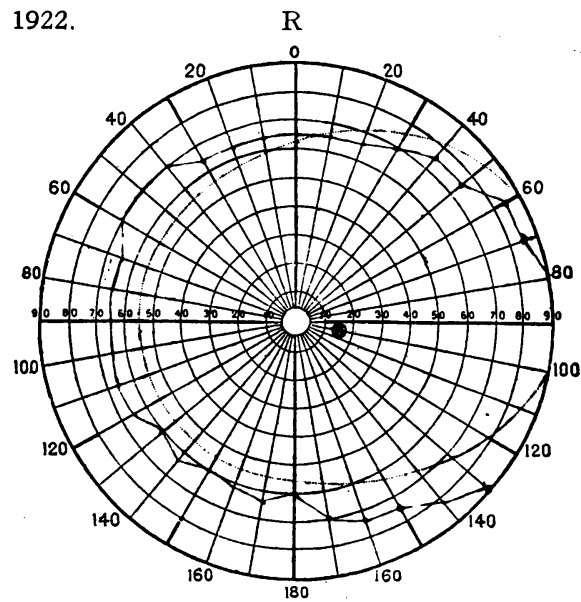

Visual Acuity-6/6. 